\title{
High temporal and spatial resolution reflectometry investigations of type-I ELMs induced edge barrier collapse at ASDEX Upgrade
}

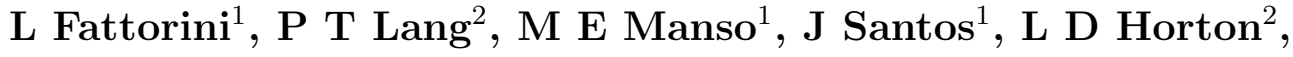 \\ G D Conway ${ }^{2}$ and the ASDEX Upgrade Team ${ }^{2}$ \\ 1 Associação EURATOM/IST, Instituto de Plasmas e Fusão Nuclear, Instituto \\ Superior Técnico, Av. Rovisco Pais, P-1049-001 Lisboa, Portugal \\ 2 Max-Planck-Institut für Plasmaphysik, EURATOM Association, Boltzmannstr. 2, \\ D-85748 Garching, Germany \\ E-mail: luca@ipfn.ist.utl.pt
}

\begin{abstract}
The edge barrier collapse induced by spontaneous and pellet triggered ELMs has been characterized by means of a new technique based on the reflectometry group delay. The analysis of the behaviour of the density layers at the plasma edge revealed small differences between the two types of ELMs. With this technique we are able to detect profile steepening and fuelling effects due to pellet injection, mainly at the high field side. The presence of asymmetries between high and low field side in the ELM dynamics has been also studied by evaluating the ELM onset time on both sides. The ELM onset on the HFS is observed to be only slightly delayed compared to the LFS onset - the barrier collapses are nearly coincident within the diagnostic time resolution. In our new findings, which apply to both spontaneous and triggered ELMs, there is no clear evidence of long HFS/LFS delays as previously observed for spontaneous ELMs.
\end{abstract}

PACS numbers: 52.70.Gw, 52.55.Rk

\section{Introduction}

The high-confinement mode (H-mode) is foreseen as the basic operation scenario for ITER. However, it is characterized by transient edge localized modes (ELMs) that cause periodic expulsions of plasma energy and particles. In particular type-I ELMs can generate large energy losses from the plasma, which may lead to unacceptably high power loads on vessel components in ITER or a fusion reactor (Federici 2006). In ASDEX Upgrade experimental studies are being carried out to limit the ELM energy. The approach to control the ELM energy is to increase the ELM frequency $f_{\text {ELM }}$ above the normal value $f_{\text {ELM }}^{0}$ ('ELM pace making'). This was motivated by the empirical finding that the ELM energy is reduced with the increase of the ELM frequency. The observation is valid both for events occouring in a naturally ELMing phase, called spontaneous ELMs, and for events caused by the pellet perturbation, called triggered 
ELMs (Lang et al 2004). The most successful method for reliable and persistent ELM control employed so far is the injection of small cryogenic D pellets, which can enhance and finally control the ELM frequency. Enhancement of $f_{\mathrm{ELM}}$ is achieved for pellet injection frequency $f_{\mathrm{Pel}}$ still below $f_{\mathrm{ELM}}^{0}$ with a mixture of spontaneous and externally triggered ELMs during the control phase. Once $f_{\mathrm{Pel}} \approx 1.5 f_{\mathrm{ELM}}^{0}$ is reached, full control with $f_{\mathrm{ELM}}=f_{\mathrm{Pel}}$ is obtained (for the currently highest available pellet rate, a maximum enhancement of $f_{\mathrm{ELM}} \approx 2.5 f_{\mathrm{ELM}}^{0}$ is achieved). This ELM pacing technique is also regarded as a promising tool for ELM physics investigations despite the impact on the confinement (Lang et al 2005a). The mild confinement reduction can be attributed to the additional pellet fuelling and is expected to be reduced once optimized small pellet injection is possible (Lang et al 2004).

However, the requirement to scale up and eventually even optimize a suitable pacing tool for larger tokamaks like ITER calls for a better understanding of the triggering process and hence the ELM itself. Current ELM models (Connor et al 1998, Wilson et al 2000) describe the periodic appearance of the ELM events under quasi steady state conditions as a limit cycle of the plasma pressure and current density in the steep gradient zone at the plasma edge encountering repeatedly a peeling-ballooning instability. Perturbation experiments like pellet induced ELM triggering do probe the response of the ELM cycle to abrupt and short time scale changes of parameters thought essential for the ELM dynamics. This way, such experiments can challenge the existing models and also help to refine them. In this context, the present study on an aspect of the dynamics involved aims to improve the understanding of the ELM process and the potential of any approaches towards its control.

Reflectometry analyses of pellet injection experiments performed so far (Lang et al 2006) were focused on the study of density profiles in the phase between ELMs, where profiles are not distorted by the ELM activity. When comparing spontaneous and triggered ELMs phases, no significant differences were found. In order to further characterize the ELM behaviour, the previous findings have been extended to the ELM event, analyzing, with the fastest measurement capability available, the density evolution in short $(\sim 2 \mathrm{~ms})$ time windows centered on the ELM events.

This investigation is focused on the characterization of the dynamics of the edge density barrier collapse due to ELMs, to find possible differences between spontaneous and triggered ELMs and asymmetries between high field side (HFS) and low field side (LFS) at ASDEX Upgrade. In this way it is hoped to gain more insight into the mechanism and the dynamics of the triggering process. Reflectometry, due to its high temporal and spatial resolution, is a diagnostic specifically suited for this type of study, allowing simultaneous probing of the plasma on the HFS and LFS. As during ELMs strong perturbations are usually present at the plasma edge, profile evaluation can result in difficulty applying standard data analysis techniques (Varela et al 2006). This situation could be especially encountered during the phase of outward particle and energy flux indicated by the peak of the $\mathrm{D}_{\alpha}$ signal at the divertor. A new technique, the Group Delay Spectrogram Slices (GDSS) technique, has been developed to visualize 
in a simple and direct way the local profile changes without the need to evaluate the density profile. This technique (Fattorini et al 2005) is based on a technique previously introduced by Manso (Manso et al 1997) and is here applied to the analysis of the density effects caused by the ELM dynamics, giving the time evolution of group delay spectrograms slices taken at selected density layers. During the last eight years the minimum reflectometry sweep repetition period has decreased from $\sim 120 \mu \mathrm{s}$ to $35 \mu \mathrm{s}$, primarily due to a factor of four reduction of the sweep duration from $100 \mu \mathrm{s}$ to $25 \mu \mathrm{s}$. This allows to study a complete ELM cycle using consecutive measurements from a single ELM, avoiding the need to collate information from several ELMs into one 'typical' ELM (Manso et al 1997). The available system memory has also increased by a factor of four allowing a longer acquisition time window.

Reflectometry studies on the LFS pedestal and scrape off layer (SOL) density profile dynamics during spontaneous ELMs have been performed, using different techniques, on several devices including JT-60U (Oyama et al 2001) and DIII-D (Zeng et al 2004, Zeng et al 2005). In the work on the characterization of spontaneous type-I ELMs on ASDEX Upgrade by Nunes et al (2004) both HFS and LFS density profile collapse have been analyzed using the DENSITY LAYER technique here described in section 3.2.

This paper is organized as follows. After a short description of the experimental set-up in the ASDEX Upgrade tokamak, section 3 reviews the techniques used to analyze the density variations from swept signals. Section 4 presents experimental results of the analysis study of density layer changes due to spontaneous and triggered ELMs. Concluding remarks are given in section 5 .

\section{Hardware}

\subsection{ASDEX Upgrade and pellet injector}

Experiments were conducted on the ASDEX Upgrade tokamak (ASDEX Upgrade Team 2003): major radius $R_{0}=1.65 \mathrm{~m}$, minor plasma radius $a_{0}=0.5 \mathrm{~m}$, plasma volume $V_{\text {Plasma }} \approx 13 \mathrm{~m}^{3}$. For the data analyzed in this paper, the tokamak was equipped with the divertor configuration DIV IIb (Neuhauser et al 2003). A typical plasma cross section is shown in figure 1. All experiments presented here were performed in lower single null configuration with a plasma current $I_{\mathrm{p}}=1 \mathrm{MA}$.

Pellets were injected via a 'looping' system launching them from the HFS upper divertor region (see figure 1) at a speed of $1000 \mathrm{~ms}^{-1}$ (Lang et al 2003). At this velocity initially cubic pellets (side length $1.4 \mathrm{~mm}$ ) are abraded to thin plates containing about $(0.18-0.25) \cdot 10^{20} \mathrm{D}$ at plasma injection. The pellet frequency is preset and injection is possible at frequencies $f_{\mathrm{Pel}} \leq 83 \mathrm{~Hz}$. With these operational parameters, shallow pellet penetration is obtained. Diodes detecting the $\mathrm{D}_{\alpha}$ ablation radiation from the plasma region including the pellet path yield a monitor signal for the pellet ablation. A fast framing camera system records pellet penetration and allows a reconstruction of the pellet trajectory. In this way, temporal correlations between pellet ablation and 


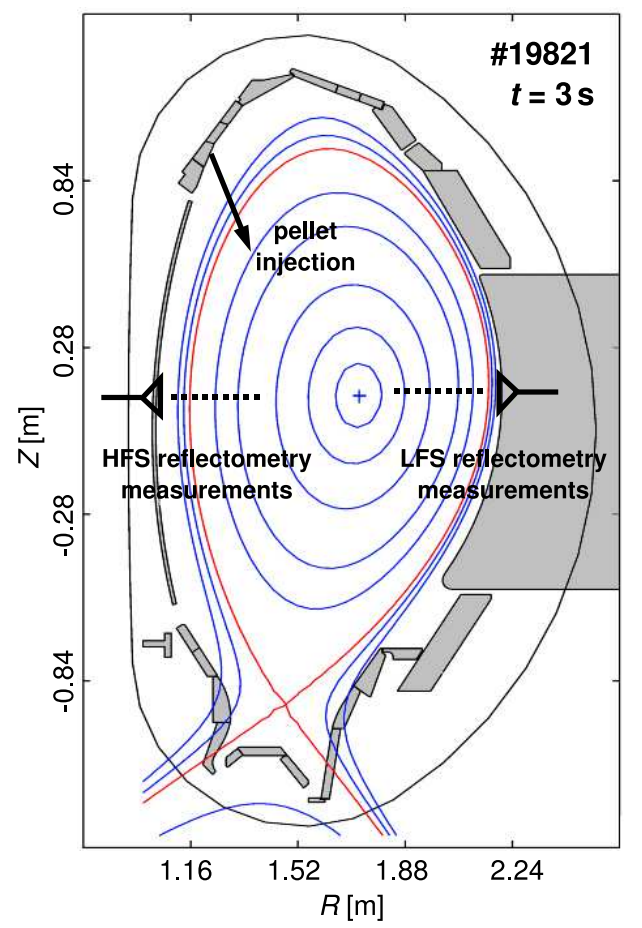

Figure 1. Typical ASDEX Upgrade cross-section (\#19821). Schematic of the lines of view of the reflectometry system at the HFS and the LFS and localization of the HFS pellet injection.

ELM onset can be related to the spatial localization of the according seed perturbation (Kocsis et al 2007).

\section{2. $F M-C W$ reflectometer}

The reflectometry basic principle is in the total reflection of an electromagnetic wave (in the millimeter range) at the cutoff layer. In O-mode, where the wave vector is orthogonal to the magnetic field and the wave electric field is parallel to the magnetic field, the relationship between the cutoff density $n_{\mathrm{ec}}$ and the probing frequency $F$ is given by this simple equation (Ginzburg 1964):

$$
n_{\mathrm{ec}}=\frac{\varepsilon_{0} m_{\mathrm{e}}(2 \pi F)^{2}}{\mathrm{e}^{2}}
$$

After propagation into the plasma, up to the cutoff layer $n_{\mathrm{ec}}$ and back, the reflected wave has a phase shift $\varphi$ relative to the reference signal, which is "equivalent" to a time delay $\tau$, the so called group delay:

$$
\tau(F)=\frac{1}{2 \pi} \frac{\mathrm{d} \varphi(F)}{\mathrm{d} f}=f_{\mathrm{b}}(F)(\mathrm{d} f / \mathrm{d} t)^{-1}
$$

that is given by the derivative of the phase shift or, equivalently, by the beat frequency $f_{\mathrm{b}}$ (frequency of the interference signal between the reflected and the reference signals) multiplied by the inverse of the sweep frequency rate $\mathrm{d} f / \mathrm{d} t$. It should be noted that $\tau(F)$ depends on the density profile $n_{\mathrm{e}}(r)$ up to the reflection. In any case, the contribution 
to $\tau(F)$ is mainly given by the density layers close to the cutoff and less by the density layers along the line of sight (Budden 1988, Swanson 1989). From the computation of $\tau$ for all frequencies inside the range of interest, using swept frequency operation, the cutoff layer position $r_{\mathrm{c}}$ (corresponding to $F$ ) is derived by the Abel inversion:

$$
\begin{aligned}
r_{\mathrm{c}}(F) & =\frac{c}{\pi} \int_{0}^{F} \mathrm{~d} f \tau(f)\left(F^{2}-f^{2}\right)^{-1 / 2} \\
& =\frac{c}{\pi}[\underbrace{\int_{0}^{F_{1}} \mathrm{~d} f \tau(f)\left(F^{2}-f^{2}\right)^{-1 / 2}}_{\text {initialization }}+\int_{F_{1}}^{F} \mathrm{~d} f \tau(f)\left(F^{2}-f^{2}\right)^{-1 / 2}]
\end{aligned}
$$

The group delay curve $\tau(F)$ is obtained by applying a sliding FFT to the reflected signal and locating the spectral peak with a best path algorithm (Varela et al 1999). The density profile $n_{\mathrm{e}}(r)$ is then reconstructed from $F$ and $\tau(F)$ using (1) and (3). O-mode can only be used above $F_{1} \approx 18 \mathrm{GHz}$ (corresponding to the initial density $n_{\mathrm{e} 1} \approx 0.45 \cdot 10^{19} \mathrm{~m}^{-3}$ ), because for lower frequencies the probing wavelength becomes too large when compared with the local density gradient scale length (the conditions given by Ginzburg are valid when the variation of the refractive index is small within a wavelength). In order to include the effect of densities below $n_{\mathrm{e} 1}$ on the group delay, it is necessary to use $\mathrm{X}$-mode propagation, where both the wave vector and the wave electric field are orthogonal to the magnetic field, or to complement the O-mode data with an initialization procedure (Varela et al 1995).

The ASDEX Upgrade FM-CW reflectometry system (Silva et al 2005) has 9 swept channels, 5 (K [18-25 GHz], Ka [25-35 GHz], Q [35-50 GHz], V [50-72 GHz] and W [72-100 GHz] bands) at the LFS and 4 (K, Ka, Q and V bands) at the HFS, probing the plasma simultaneously close to the equatorial plane (see figure 1). Antennae are installed in the same toroidal sector where the pellet injector is located. All channels can be operated either in swept frequency mode (broadband frequency) or in fixed frequency mode. The group delay, and consequently the density profile, is computed when operating in swept mode, where the probing frequency $F$ is swept in $25 \mu$ s over the all bands frequency ranges with a minimum sweep repetition period of $35 \mu \mathrm{s}$. In fixed frequency mode operation it is possible to study density fluctuations with a sampling rate up to $1 \mu \mathrm{s}$. Two more channels at the LFS, FLQ (Q band) and FLV (V band), operating in fixed frequency mode with a 1 or $2 \mu$ s sampling rate are used as fluctuation

monitors. X-mode measurements were not available in this experimental campaign and density profiles were initialized using a linear approximation of the group delay curve, assuming a fixed position $r_{0}$ for the first plasma layer (at zero density). Although this procedure introduces some errors, these are significant close to $n_{\mathrm{e} 1}$ but decrease for higher densities (Varela et al 1995).

\section{Analysis techniques}

The investigations reported in this paper have been carried out applying the GDSS technique. The evaluation of the ELM onset time at HFS and LFS is necessary for the 
comparison with other diagnostics (see section 4.3) and to detect eventually asymmetries between HFS and LFS in the barrier collapse (see section 4.4). In order to validate the accuracy of the GDSS technique in this evaluation, the GDSS analysis has been checked against the DENSITY LAYER analysis. These two techniques are described in the following sections.

It should be noted that the computation of the cutoff layer position $r_{\mathrm{c}}(F)$ (3) requires one additional integration process compared with the computation of the group delay $\tau(F)(2)$. The GDSS technique is thus in principle more sensitive to density variations due to the ELM occurrence than the DENSITY LAYER. In the latter the radial displacement of a certain density $n_{\mathrm{ec}}$ is computed integrating the group delay curve from zero up to the corresponding frequency $F=(e / 2 \pi) \sqrt{n_{e c} / \varepsilon_{0} m_{\mathrm{e}}}$. This smooths the variation, due to the density layer movement, in the group delay.

\subsection{The Group Delay Spectrogram Slices (GDSS) technique}

The spectrogram obtained from the reflected broadband signal (Santos et al 1997) gives the beat frequency energy distribution $f_{\mathrm{b}}$ vs. the probing frequency $F$ (or plasma density). As shown in (2), the main beat frequency line $f_{\mathrm{b}}(F)$ allows calculating the group delay data $\tau(F)$ necessary for evaluating the distances from the antenna to the reflecting layers, $r\left(n_{\mathrm{e}}\right)$, by using (3). The spectrogram shows that the energy at each window of analysis can be scattered to beat frequency locations away from the main line $f_{\mathrm{b}}(F)$. The scattered energy and the local deviations of the energy from the main line $f_{\mathrm{b}}(F)$ indicate, respectively, either the presence of fluctuations or movements of the reflecting layer (or in the propagation path). For the density profile evaluation, the best path algorithm is used to extract the mean evolution of $f_{\mathrm{b}}(F)$ line in order to calculate the distance data $r\left(n_{\mathrm{e}}\right)$. In cases where this procedure is not sufficient, a reliable density profile cannot be obtained - this usually occurs when the $\tau(F)$ is highly distorted. An example, where the density profile can be reconstructed only up to $F_{\alpha}$, is shown in figure 2 . However, even in cases where it is not possible to derive a density profile, the spectrogram contains information on density perturbations and localized movements.

From a sequence of consecutive spectrograms, i.e. sweeps, the time evolution of the spectral content in windows of analysis centered at chosen fixed probing frequencies can be extracted. A schematic representation of the GDSS technique is shown in figure 3. From each one of a set of $N$ consecutive group delay spectrograms we extract a vertical spectrogram slice centered at a chosen frequency $F^{*}$. The resulting slice is a one-dimensional profile of the group delay energy distribution at the frequency $F^{*}$, computed using an FFT on appropriate frequency interval $\Delta F$ centered at $F^{*}$. The collection of these $N$ slices (from now on designated as 'GDSS plot') represents the temporal evolution of the group delay spectral content for the plasma density $n_{\mathrm{e}}^{*}$ corresponding to $F^{*}$.

This technique has two main advantages: i) it provides local inward or outward 


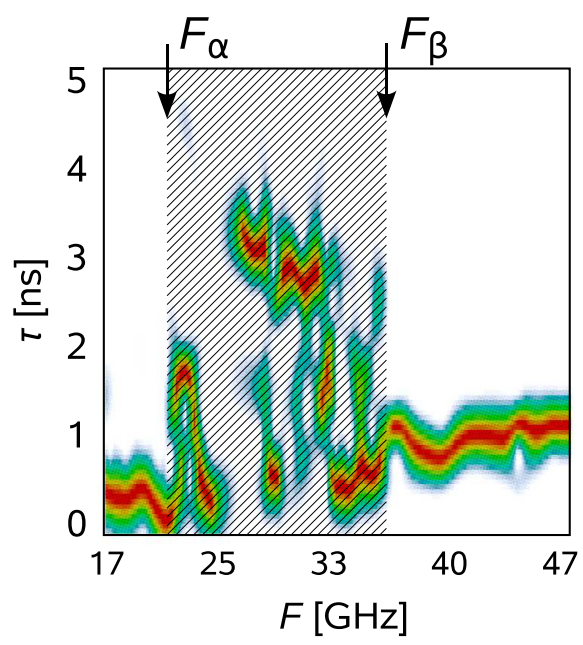

Figure 2. Group delay spectrogram example where part of the frequency range of the reflected signal is highly distorted. While the density profile can be reconstructed only up to $F_{\alpha}$ the GDSS technique can be applied on both unperturbed regions $F<F_{\alpha}$ and $F>F_{\beta}$.

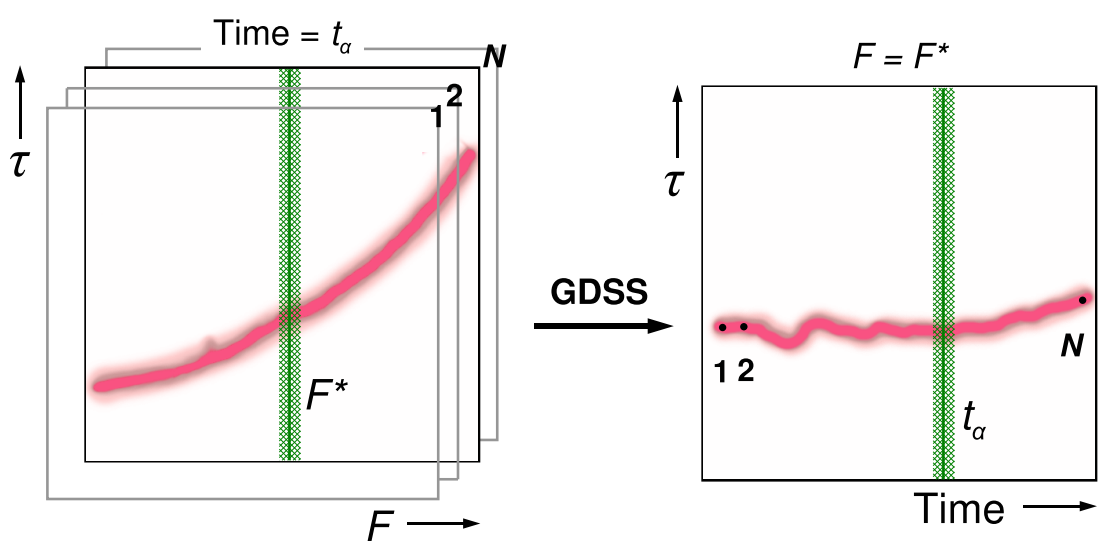

Figure 3. The basic idea of the GDSS technique: the temporal evolution of the group delay spectrogram slice (green) centered on a certain frequency $F^{*}$ is extracted from a set of $N$ consecutive spectrograms.

density layer movements even in the case where a density profile cannot be obtained (the radial amplitude of those plasma movements, however, cannot be extracted) - as for the density layers corresponding to $F>F_{\beta}$ shown in the example in figure 2 that can be now analyzed; ii) it is very sensitive to local profile changes and does not depend on errors caused by the integration needed to calculate the distance (Abel inversion) or by the initialization procedure used for the profile evaluation.

For a correct interpretation of the movement of a single density layer, it is anyway necessary to simultaneously look at several GDSS plots (corresponding to different density layers). If in a certain time window, for example, $\tau\left(F_{1}\right)$ and $\tau\left(F_{2}\right)$ are constant while $\tau\left(F_{3}\right)$ is increasing (where $F_{1}<F_{2}<F_{3}$ ), we can deduct that the density layer 
corresponding to the probing frequency $F_{3}$ is moving inward. Instead, simply looking at one single GDSS plot could result in a wrong observation, as would happen when the probing frequency $F$ is located at the pivot point of a gradient collapse - during an ELM event the density layer at the pivot point is not moving, while the group delay is slightly increasing due to the outward movement of the outer layers.

The spectrograms are obtained with the simultaneous operation of the different swept channels. In order to have the best time resolution, all channels were operated in swept mode at the minimum sweep repetition period of $35 \mu$ s (sweep duration $25 \mu \mathrm{s}$, interval between sweeps $10 \mu \mathrm{s}$ ), corresponding to a total data acquisition window of $107.24 \mathrm{~ms}$ with 3066 sweeps acquired (limitation imposed by the present system memory).

3.1.1. A Group Delay Spectrogram Slices example To illustrate the technique, we present an example concerning the temporal evolution of a chosen density layer during an ELM. Swept reflectometry measurements have been performed in the time window $t=3.025515-3.026740 \mathrm{~s}$, for the ASDEX Upgrade H-mode discharge \#19821, that contains a spontaneous type-I ELM having onset time $t_{0, \mathrm{D}_{\alpha}}=3.025790 \mathrm{~s}$, evaluated from the outer divertor $\mathrm{D}_{\alpha}$ signal (in the following the outer divertor $\mathrm{D}_{\alpha}$ signal will be indicated by ' $\mathrm{D}_{\alpha}$ signal', except where differently specified). To study the density layer $n_{\mathrm{e}}=4.3 \cdot 10^{19} \mathrm{~m}^{-3}$, group delay spectrograms have been computed for all the thirty-six sweeps acquired in the time window. An example of three of the HFS spectrograms for $t=3.025725 \mathrm{~s}\left(t^{\prime}=-65 \mu \mathrm{s}\right.$, where $\left.t^{\prime} \equiv t-t_{0, \mathrm{D}_{\alpha}}\right), t=3.026145 \mathrm{~s}\left(t^{\prime}=+355 \mu \mathrm{s}\right)$ and $t=3.026600 \mathrm{~s}\left(t^{\prime}=+810 \mu \mathrm{s}\right)$ are displayed in figure $4(\mathrm{a})$, left column - the slice of the spectrogram at the selected frequency $F=59 \mathrm{GHz}$ (equivalent to the density $n_{\mathrm{e}}=4.3 \cdot 10^{19} \mathrm{~m}^{-3}$ ) is marked in red. The reconstruction of the corresponding density profiles requires, as described in section 2.2, the computation of the group delay curves by applying the best path algorithm to the group delay spectrograms. The computed group delay curves for $t^{\prime}=-65 \mu \mathrm{s}, t^{\prime}=+355 \mu \mathrm{s}$ and $t^{\prime}=+810 \mu \mathrm{s}$ are depicted in figure 4(a), right column, and the corresponding density profiles are shown in figure 4(b), where the analyzed density layer is also indicated by a horizontal red line. When we want to study the displacement of a specific density layer, the GDSS technique lets us avoid calculating the density profiles that, as mentioned before, sometimes cannot be extracted when strong fluctuations are present. The GDSS, moreover, maintains the information about the level of fluctuations at the specific density layer. The temporal evolution of the group delay spectrogram slices ('GDSS plot') for $n_{\mathrm{e}}=4.3 \cdot 10^{19} \mathrm{~m}^{-3}$ obtained from thirty-six sweeps around the ELM is represented in figure 4(c) together with the outer divertor $\mathrm{D}_{\alpha}$ signal trace. The response of the selected plasma layer to the onset of the spontaneous ELM at $t_{0, \mathrm{D}_{\alpha}}=3.025790 \mathrm{~s}$ can be clearly seen from the increase of the group delay starting at $t^{\prime}=+75 \mu \mathrm{s}(t=3.025865 \mathrm{~s})$. This indicates the inward movement of the density layer (away from the launching-receiving antenna, located at $R=0.987 \mathrm{~m}$ ) and therefore a density profile flattening caused by the ELM. In figure 4(c) a mild increase of the fluctuation level after the ELM onset till $t^{\prime}=+355 \mu$ s and again 


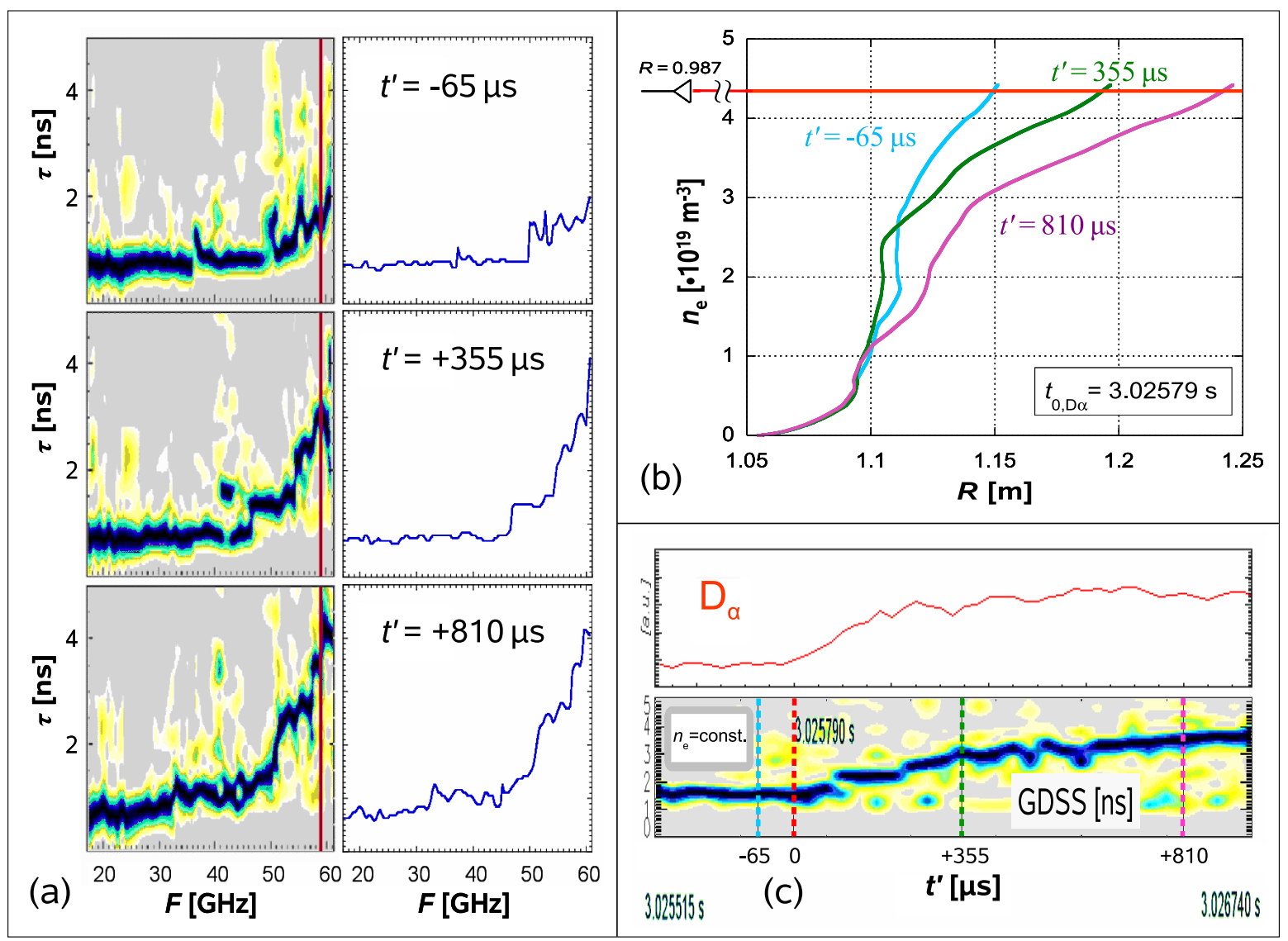

Figure 4. (a) Group delay spectrogram examples, and respective best path group delay curves, for three HFS sweeps at relative time difference with respect the ELM onset time $t_{0, D_{\alpha}} t^{\prime}=-65 \mu \mathrm{s}, t^{\prime}=+355 \mu \mathrm{s}$ and $t^{\prime}=+810 \mu \mathrm{s}(\# 19821)$ — the vertical red lines indicate the group delay spectrogram slices for the analyzed density $n_{\mathrm{e}}=4.3 \cdot 10^{19} \mathrm{~m}^{-3}$, corresponding to $F=59 \mathrm{GHz}$. (b) HFS density profiles — the horizontal red line indicates the analyzed density; the four HFS emitting/receiving antennae are located at $R \approx 0.99 \mathrm{~m}$. (c) Temporal evolution of the HFS GDSS at $F=59 \mathrm{GHz}$ and $\mathrm{D}_{\alpha}$ signal - temporal position of sweeps $t^{\prime}=-65 \mu \mathrm{s}$ (light blue), $t^{\prime}=+355 \mu \mathrm{s}$ (green) and $t^{\prime}=+810 \mu \mathrm{s}$ (purple) are indicated by vertical dashed lines, as well as the ELM onset time (red).

in the recovery phase around $t^{\prime}=+810 \mu$ s can also be observed.

\subsection{The density layer radial displacements (DENSITY LAYER) technique}

The technique based on the the radial displacements of density layers, the DENSITY LAYER technique, extracts the radial displacement $r_{n_{\mathrm{e}}^{*}}$ of a certain chosen density layer $n_{\mathrm{e}}^{*}$ from the reconstructed density profiles. Density profiles are computed from the group delay spectrograms, necessary for the computation of the GDSS, as described in section 2.2. Plotting the temporal evolution of the radial displacements for several layers, it is possible to detect the ELM onset time and the pellet ablation time (see section 4.2). In figure 5 an example for a spontaneous and a triggered ELM from the data set that will be introduced in section 4.3 is shown. The ELM onset corresponds 


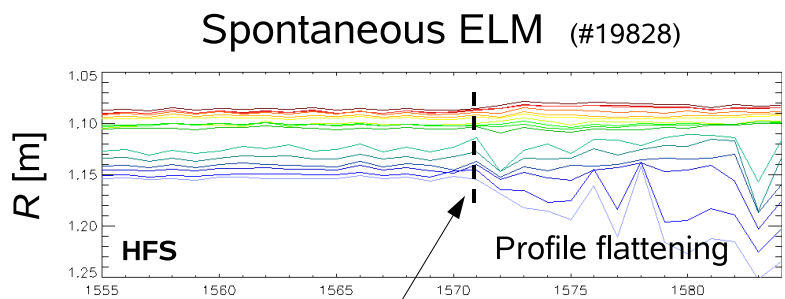

Triggered ELM (\#19821)
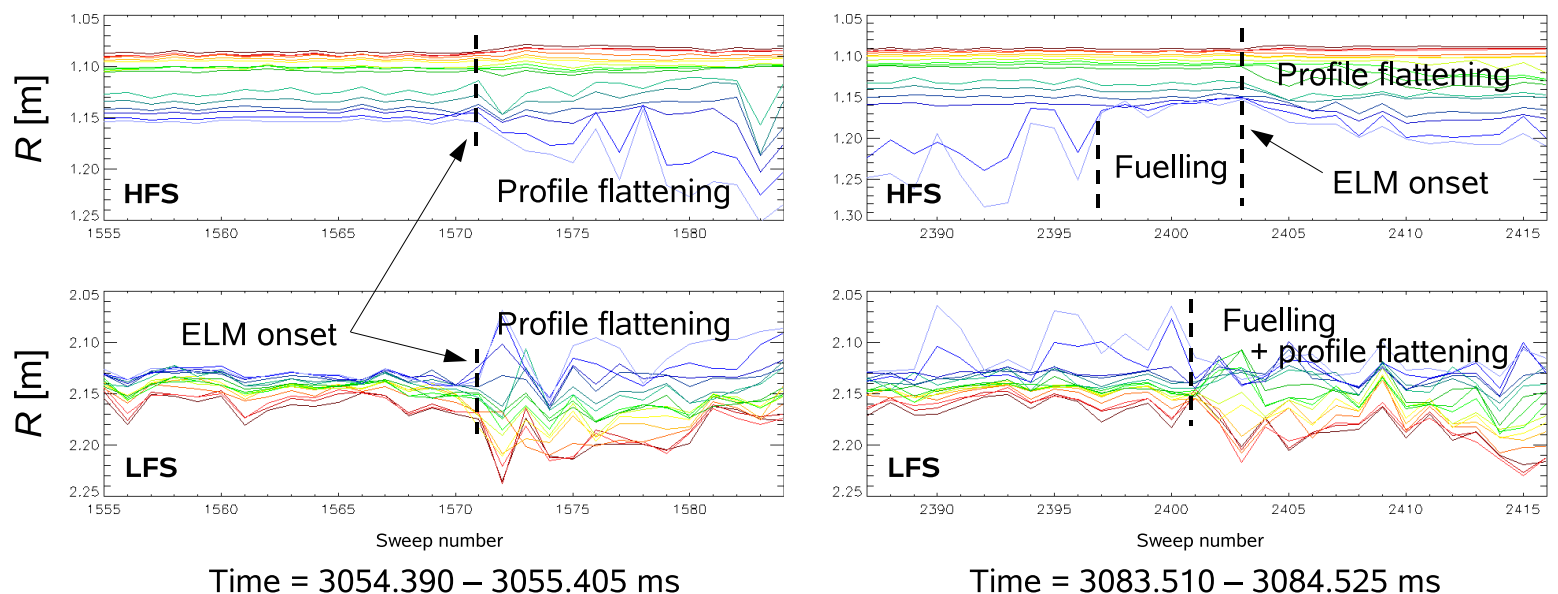

Time $=3083.510-3084.525 \mathrm{~ms}$

Figure 5. DENSITY LAYER technique example for spontaneous and triggered ELM. Up to sixteen density layer radial displacements are plotted (density increases from dark red to light blue). ELM onset time and pellet ablation time are indicated by dashed vertical lines.

to the temporal position where the constant density curves start to spread radially, representing the beginning of profile flattening. Regarding the pellet triggered ELMs, plotting the radial displacement of the closest frequencies above the highest reflected frequency, one can also detect the ablation time. For these frequencies the respective computed radial positions display a large scatter because the density is not high enough (or the density profile is not steep enough). The ablation time corresponds to the time where the scattering of points on the curve starts to disappear due to the density increase.

In the DENSITY LAYER technique, each curve in the plots $r_{n_{\mathrm{e}}}$ vs. $t$ (see figure 5 ), corresponding to a certain density $n_{\mathrm{e}}^{*}$, contains all information on the group delay of lower densities $n_{\mathrm{e}} \leq n_{\mathrm{e}}^{*}(3)$. In the density and/or temporal regions where the density profile is highly distorted by turbulence, the points in the radial displacement curve $r\left(n_{\mathrm{e}}, t\right)$ exhibit a large scatter. Concerning the fixed position for the first plasma layer $r_{0}$ (see section 2.2), it should be noted that, due to this assumption, the radial displacements obtained with the DENSITY LAYER technique do not exactly represent the real values, especially at the very edge. This does not in any way affect the detection of the ELM onset (our main aim) because, as described at the end of section 2.2, only the movements of the layers at the very edge are affected (specifically by becoming shorter during an ELM event). Moreover, as also shown in Zeng et al (2004), the larger outward movement of the very edge plasma layers occur after the ELM onset, precisely at the ELM crash (i.e. at the peak of the $\mathrm{D}_{\alpha}$ signal). 


\section{Results}

The main aim of this study is to examine HFS/LFS asymmetry for pellet triggered ELMs and spontaneous ELMs and to compare the results with previous findings for spontaneous ELMs (Nunes et al 2004) in the hot edge type-I regime characterized by a fast ELM onset (Kocsis et al 2007). To provide stable and robust target plasmas with well separated spontaneous ELM events, such investigations were usually performed in the perturbative limit, where the pellet rate was small compared to the spontaneous ELM repetition frequency and also small enough to avoid any lasting pellet impact on the global plasma parameters.

Before showing the statistical analysis for the evaluation of the ELM onset time (section 4.3) and HFS/LFS asymmetries (section 4.4), an overview on spontaneous and triggered ELM dynamics is presented.

\subsection{Spontaneous vs. triggered ELM behaviour}

To analyze and to compare spontaneous and pellet triggered ELMs at the HFS we can refer to a typical experiment with a mixture of spontaneous and triggered ELMs as in the ASDEX Upgrade H-mode discharge \#19821 (plasma current $1 \mathrm{MA}$, average density $n_{\mathrm{e}}=8.8 \cdot 10^{19} \mathrm{~m}^{-3}$ ). For this discharge pellets were launched from the HFS between 1.4 and $3.4 \mathrm{~s}$ with $5 \mathrm{~Hz}$ frequency and $240 \mathrm{~ms}^{-1}$ velocity. There is no significant change in the plasma parameters due to the pellet injection since $f_{\mathrm{Pel}} \ll f_{\mathrm{ELM}}^{0}$. In the following, results are presented that were obtained by analyzing two $2 \mathrm{~ms}$ time windows centered respectively at a spontaneous and a triggered ELM event with very similar plasma parameters. In the phase between ELMs, where profiles are not perturbed by the ELM activity, the pedestal top density is $\approx 5.2 \cdot 10^{19} \mathrm{~m}^{-3}$. For the spontaneous ELM (see figure 6(a), lower part) a clear inward movement is seen at plasma layers $n_{\mathrm{e}}=3.8 \cdot 10^{19} \mathrm{~m}^{-3}$ and $n_{\mathrm{e}}=4.3 \cdot 10^{19} \mathrm{~m}^{-3}$, corresponding to probing frequencies $F=55 \mathrm{GHz}$ and $F=59 \mathrm{GHz}$, starting at the beginning of the rise of the $\mathrm{D}_{\alpha}$ signal. The red arrow indicates the temporal localization of the ELM onset evaluated from the GDSS analysis that corresponds, for this particular density, to the increase of the group delay. In the case of the pellet triggered ELM (see figure 6(b), lower part), we first notice that the movements of the plasma layers are shifted to higher densities $n_{\mathrm{e}}=5.4 \cdot 10^{19} \mathrm{~m}^{-3}$ $(F=66 \mathrm{GHz})$ and $n_{\mathrm{e}}=5.9 \cdot 10^{19} \mathrm{~m}^{-3}(F=69 \mathrm{GHz})$. We can also see that just after the pellet injection, resulting in an increase of the density at the HFS (the line integrated density from DCN interferometer rises from $5.8 \cdot 10^{19}$ to $6.2 \cdot 10^{19} \mathrm{~m}^{-3}$ ), the entire $\mathrm{V}$ band channel (50-72 GHz) comes into operation. In particular, for the frequencies $F=66 \mathrm{GHz}$ and $F=69 \mathrm{GHz}$, shown in the lower part of figure $6(\mathrm{~b})$, the high scattering of the group delay in the GDSS before the pellet injection is due to the fact that the reflected signal cannot be detected because the density is not sufficiently high, or the density gradient is not steep enough, to reflect the probing microwaves. This behaviour disappears a few tens of microseconds after the beginning of the ablation of the pellet into the plasma due to the increasing density. Later, as for the spontaneous ELM, the group delay starts 
Spontaneous ELM

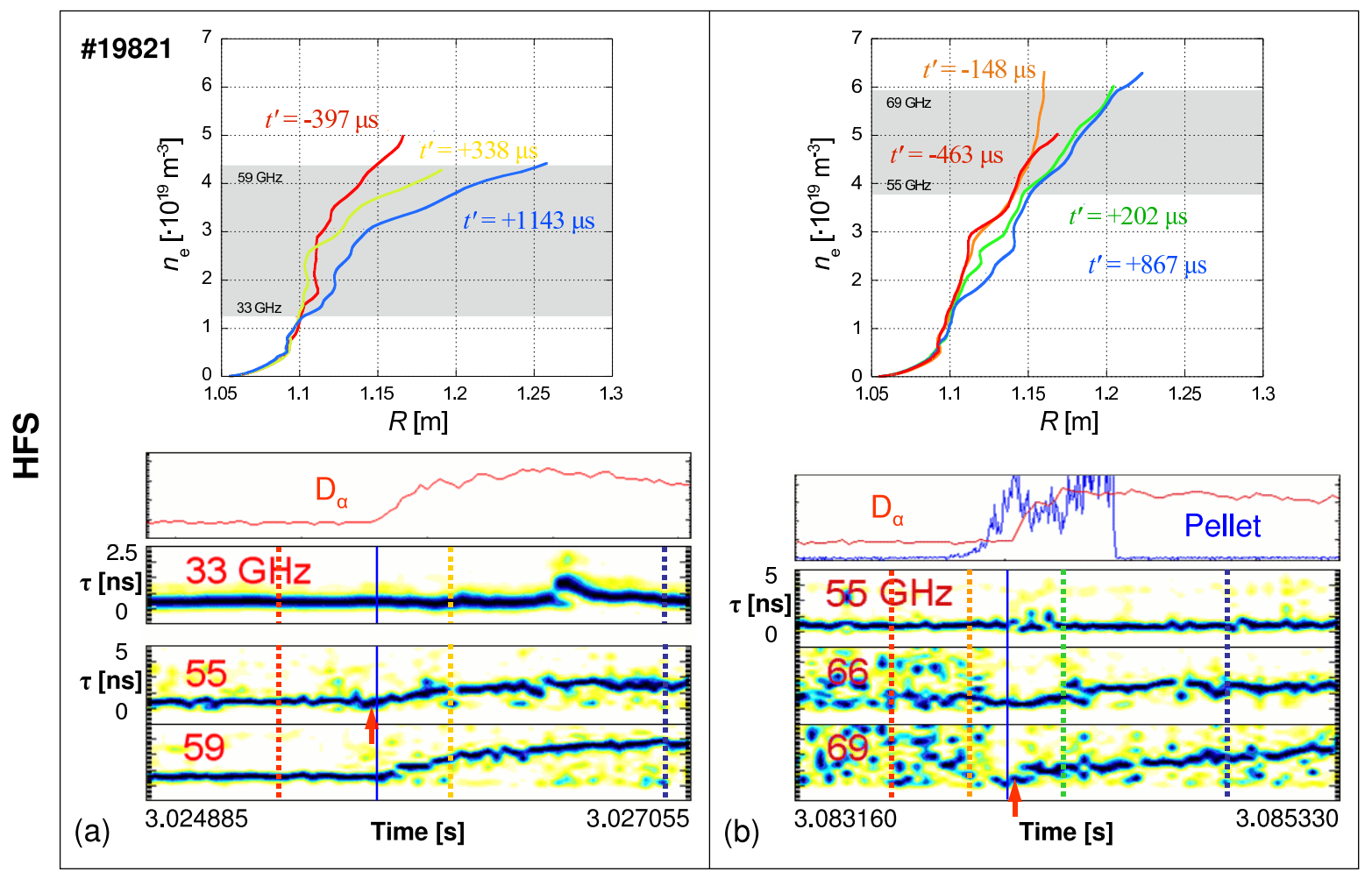

Triggered ELM

Figure 6. Temporal evolution of the HFS GDSS (\#19821), at three frequencies, for (a) a spontaneous ELM and (b) a triggered ELM. Also shown are HFS density profiles at the times marked in the GDSS plots by same color dashed lines, a divertor $\mathrm{D}_{\alpha}$ trace showing the ELM onset and, for the triggered ELM, the pellet ablation radiation signal. Vertical blue lines indicate the MHD ELM onset (see section 4.3), $t^{\prime}$ values refer to this time marker.

to increase from the beginning of the rise of the $\mathrm{D}_{\alpha}$ signal. These observations match (see also section 4.4 for a quantitative evaluation) the early HFS ablation time, $\approx 70 \mu \mathrm{s}$ before the MHD time marker (see section 4.3 ), and the $\approx 100 \mu$ s between the start of the pellet ablation and the ELM onset as shown in Kocsis et al (2007). The GDSS plots also show how the density profile continues to flatten after the peak of the $\mathrm{D}_{\alpha}$ signal. In the upper parts of figure 6(a) and 6(b) the density profiles at HFS corresponding to the main ELM phases are depicted (temporal localization of the density profiles are indicated by same color dashed lines in the GDSS plot - grey areas represent the density ranges investigated by the GDSS analysis). The plasma density profiles are the same in the unperturbed phase (red lines), before the spontaneous ELM onset and before the pellet injection. Differently from the density profile flattening occurring for the spontaneous ELM (see figure 6(a), upper part), for the triggered ELM the density peaks just after the pellet injection (orange line in figure 6(b), upper part) and the profile is measured up to $n_{\mathrm{e}}=6.4 \cdot 10^{19} \mathrm{~m}^{-3}$. In the phase after the peak of the $\mathrm{D}_{\alpha}$ signal the profile (blue lines) is still flat but for the triggered ELM it is steeper. This is due to two effects: the flattening due to the ELM and the density fuelling due to the pellet ablation (which 
will be discussed in more detail in section 4.2).

The behaviour of the spontaneous ELM at the LFS is similar to the HFS while for the triggered ELM the fuelling effect takes a longer time compared with the HFS, resulting in a delayed ELM onset detection. Moreover, at the LFS all plasma layers are generally more turbulent after the beginning of both types of ELMs compared with the HFS. It should be noted that, on both the HFS and LFS GDSS plots for the triggered ELM, the group delay decreases for a few tens of microseconds before it begins to increase. This characteristic will be also described in the next section.

\subsection{Pellet perturbative effect vs. density fuelling}

The two main effects that can be observed from the GDSS plots are the density profile flattening, corresponding to the barrier collapse, and the density profile steepening in the upper part of the profile itself. The density profile flattening is due to the ELM occurrence (for both spontaneous and triggered ELMs), while the density profile steepening is due to the pellet injection fuelling (for triggered ELMs only). As it will be described in the following, during the pellet ablation phase it is not possible to separate completely fuelling and barrier collapse, that generate opposite effects on the density profile, but only to analyze their overall effect.

The general behaviour of spontaneous and triggered ELMs at the HFS is shown in figure 7. For the spontaneous ELMs (figure 7(a)) the group delay in the lower density layers decreases, corresponding to an outward movement of the layer (moving closer to the antenna), while for higher density layers the group delay increases, corresponding to an inward movement of the density layer (moving away from the antenna), as reported in Nunes et al (2004). For the pellet triggered ELMs (figure 7(b)) the general behaviour is very similar to the spontaneous counterpart, except that at the higher densities the group delay often decreases for at least $70 \mu \mathrm{s}$ before it starts to increase again. This decrease is due to the pellet fuelling, which steepens the density profile at the higher densities (see also orange line in figure 6(b), upper part). After this short period the group delay increases again as for the spontaneous ELMs. In our study we did not see any evidence of an inward moving density pulse at the HFS as described in Kocsis et al (2007). A possible explanation can be found taking in consideration that the effect of the pellet is more evident in the upper part of the density profile, close to end of the measured frequency range, and also that the minimum sweep repetition period (35 $\mu \mathrm{s})$ is not short enough to follow the fast pulse. At the LFS the behaviour is similar but the steepening effect is not clearly observed, except in a few cases where it is anyway much less pronounced.

The pellet density fuelling effects can be clearly observed by means of the GDSS analysis. In fact, looking at frequencies just above the maximum reflected frequency (in the phase of unperturbed plasma before the pellet injection), it is possible to detect the transition where the probing microwave begins to be reflected by the plasma. One can see from figure 8(a) that for these frequencies at HFS there is a sharp transition. After 


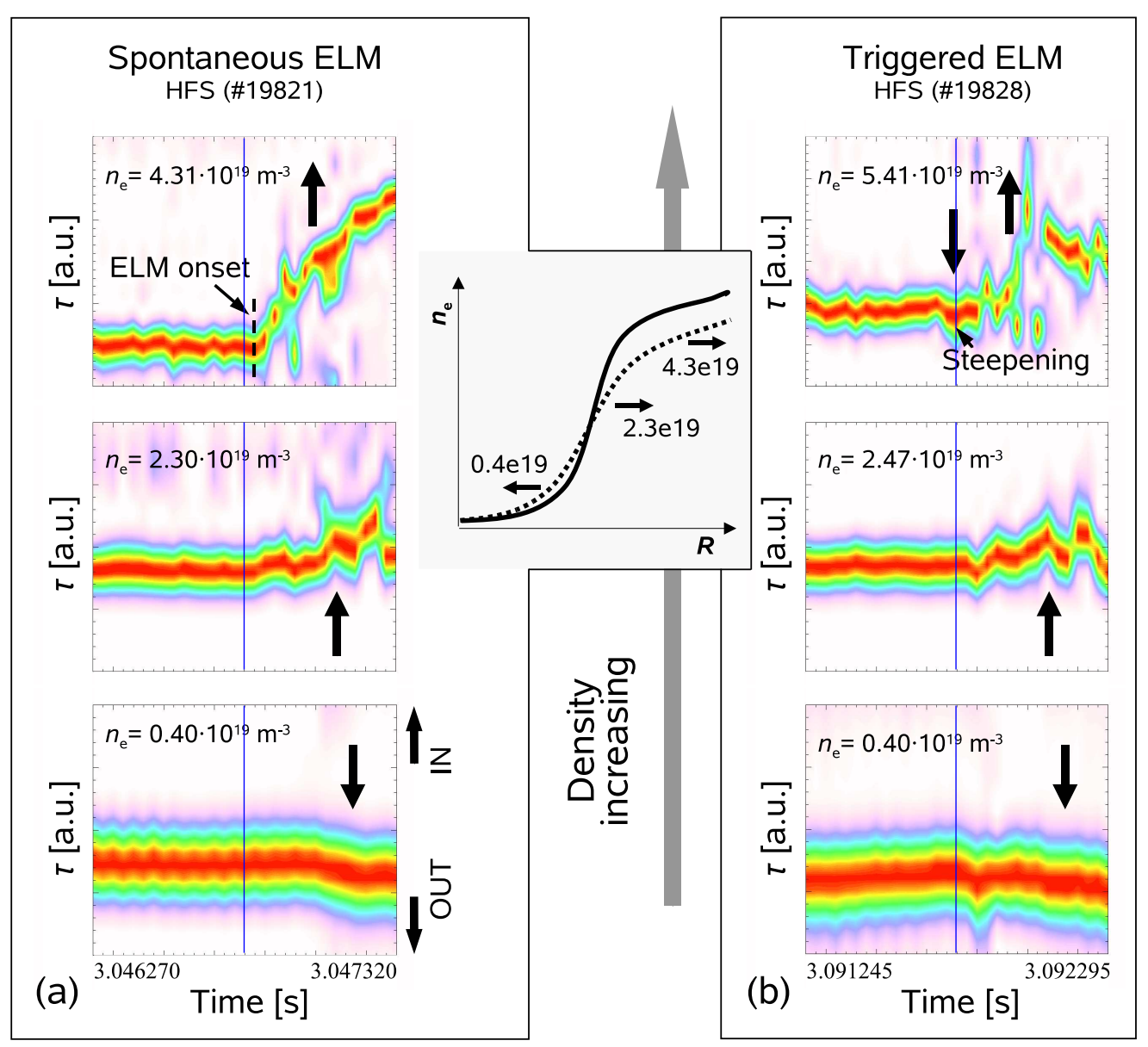

Figure 7. HFS GDSS for a (a) spontaneous (\#19821) and (b) triggered (\#19828) ELM comparison representing the main situations occurring at different densities. Black arrows indicate the movement of the plasma layer while vertical blue lines the MHD ELM onset (see section 4.3). Indicative density profile flattening for the spontaneous ELM is shown in the central box.

the transition the group delay is clearly reconstructed and starts to decrease, showing once more the steepening of the profile followed by its flattening. At the HFS it is possible to evaluate the ablation time (the time at which the pellet begins to ablate into the plasma) with good accuracy from reflectometry measurements by the temporal localization of the sharp transition (indicated by a vertical dashed line in the plots). At the LFS, the fuelling takes some time to reach the LFS. The GDSS (see figure 8(b)) show a slow transition between the large scattered area and a quite well defined group delay curve. Under these circumstances is still possible to detect an ablation time as the beginning of the decrease of the extension of the scattered area, albeit with less accuracy than the onset time calculated from the HFS data. Moreover, the LFS ablation time (negative value) can be slightly overestimated (i.e. detected later) when it is not possible to measure the highest frequency where this effect takes place. We would like to anticipate that, as will be discussed in section 4.4, the ablation time of the LFS compared with the HFS does not seem consistent with a parallel ion sound speed flux 


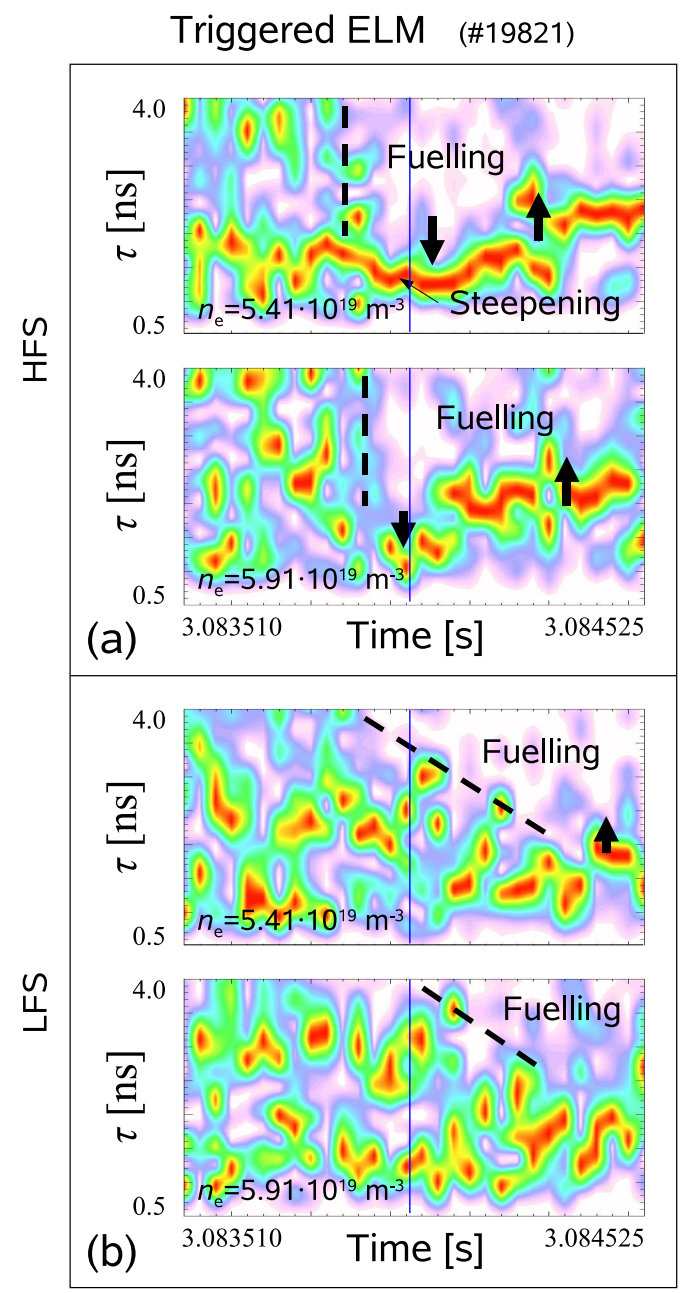

Figure 8. GDSS showing fuelling and steepening effects on high density layers at (a) HFS and (b) LFS. Dashed black lines separate the region with lower/higher density (lost/reflected microwaves). Vertical blue lines indicate the MHD ELM onset (see section 4.3)

of the pellet ablated density from the HFS to the LFS inside the closed flux surface. As a caveat, it should be noted that a rigorous evaluation is extremely difficult due to the non-instantaneous nature of the transition on the LFS.

The pellet ablation also makes the evaluation of the ELM onset time less accurate because two effects, fuelling and barrier collapse, exist simultaneously for a short period of time. The ELM onset for triggered ELMs can be slightly overestimated.

\subsection{Statistical analysis}

To analyze the general features of dynamics of the two types of ELMs and to evaluate possible asymmetries between HFS and LFS in the ELM onset time a statistical study has been carried out. A set of ELMs, 22 spontaneous and 4 pellet triggered, with a very similar plasma scenario, has been selected from four ASDEX Upgrade plasma 
discharges: \#19821, \#19828, \#19911 and \#20319. These deuterium discharges are low triangularity ELMy H-modes with $I_{\mathrm{p}}=1 \mathrm{MA}, B_{\mathrm{t}} \approx-2.7 \mathrm{~T}, q_{95} \approx 4.9, \delta_{\mathrm{av}} \approx 0.25$, $\kappa \approx 1.81$ and $n_{\mathrm{e}, \text { av }} \approx 9 \cdot 10^{19} \mathrm{~m}^{-3}$, where $I_{\mathrm{p}}$ is the plasma current, $B_{\mathrm{t}}$ the toroidal magnetic field, $q_{95}$ the safety factor calculated at the magnetic surface corresponding to $95 \%$ of the flux, $\delta_{\mathrm{av}}$ the average triangularity calculated as the average of $\delta_{\mathrm{u}}$ and $\delta_{\mathrm{l}}$ (upper and lower triangularity, respectively), $\kappa$ the elongation and $n_{\mathrm{e}, \text { av }}$ the average density. Discharge \#19911 is an exception with different toroidal magnetic field, safety factor and elongation: $B_{\mathrm{t}} \approx-2.0 \mathrm{~T}, q_{95} \approx 3.1$ and $\kappa \approx 1.66$. All discharges were heated with a neutral beam injection $(\mathrm{NBI})$ power of $P_{\mathrm{NBI}} \approx 5 \mathrm{MW}$. Two of the discharges had, in addition, ion cyclotron resonance heating: $P_{\mathrm{ICRH}} \approx 1.3 \mathrm{MW}$ (\#20319 only) and $P_{\mathrm{ICRH}} \approx 0.2 \mathrm{MW}$ (\#19828 only).

These data were selected based on the availability of adequate reflectometry data and of other diagnostics: Mirnov pick-up coil measurements, electron temperature from ECE and divertor $\mathrm{D}_{\alpha}$ - only ELMs in phases with no strong "background" MHD activity were chosen to allow for a precise detection of the ELM onset. Operating the reflectometer at the maximum acquisition rate, the maximum temporal acquisition window is limited to around $107 \mathrm{~ms}$ (due to memory limitations). Consequently, for the $10 \mathrm{~Hz}$ pellet frequency (used in these discharges), it is possible to collect only one triggered ELM in the acquisition window. All ELMs in the set have been analyzed with the GDSS technique in $1 \mathrm{~ms}$ time windows, centered at the ELM event. For the evaluation of the ELM onset time at HFS and LFS (see also section 4.4) the GDSS analysis has been validated by the DENSITY LAYER analysis. For both techniques, sixteen density layers have been selected in the density range between $n_{\mathrm{e}}=0.4 \cdot 10^{19} \mathrm{~m}^{-3}$ and $n_{\mathrm{e}}=6.0 \cdot 10^{19} \mathrm{~m}^{-3}$. For each ELM, most of the sixteen analyzed GDSS exhibit a clear variation (decreasing at low densities or increasing at high densities) of the group delay starting from a certain time $t_{j}$. The ELM onset time has been defined as the minimum of these values $t_{0} \equiv \min \left\{t_{j}\right\}_{j=1 \ldots 16}$. For the DENSITY LAYER technique the ELM onset time has been defined as the time where the radial displacement curves start to spread radially, as described in section 3.2 .

A first experimental problem to solve before performing any detailed ELM analysis is to define a characteristic event within the ELM history, which can serve as a sufficiently accurate marker to compare the time evolution of different ELMs. The onset of the MHD activity recorded by the magnetic pick-up coils turns out to satisfy this requirement properly (Lang et al 2006). At the onset of a type-I ELM the $\mathrm{d} B / \mathrm{d} t$ signal increases rapidly on all magnetic probes at any position in the torus within about $20 \mu \mathrm{s}$. A detailed analysis of the onset time at different coil locations (toroidal and poloidal) can be found in Kocsis et al (2007), showing that no coil is significantly ahead for the ELM onset. The coil onset time is statistically distributed. This can be qualitatively explained by a random ELM onset (i.e. the ELM starts at random toroidal location) and the fast shear Alfvén wave communication along magnetic field lines, transmitting the initial magnetic seed perturbation, wherever started, all around the torus. In ASDEX Upgrade the observed statistical onset delay of any of the coils with respect to a reference 
one is $0.6 \mu \mathrm{s}$ (with $\sigma=9.0 \mu \mathrm{s}$ ) for spontaneous and $0.8 \mu \mathrm{s}$ (with $\sigma=9.6 \mu \mathrm{s}$ ) for triggered ELMs (Lang et al 2006).

Given the ELM onset time marker as defined by a reference pick-up coil, we analyzed the breakdown of the transport barrier using the available fast diagnostics. Other than the magnetic probes, reflectometry is the only diagnostic available to detect simultaneously the ELM onset time at HFS and LFS. The breakdown of the transport barrier soon after the ELM MHD onset results in a drop of the edge electron temperature, an erosion of the steep density gradient and an enhancement of the density fluctuations in this region. The rapidly increasing outflux of particles and energy into the SOL and the divertor drives up the divertor radiation. The time at which a change is observed in these quantities has been statistically analyzed, results are shown in figure 9 . All plotted values refer to the $t \equiv 0$ ELM MHD onset time marker, evaluated from a

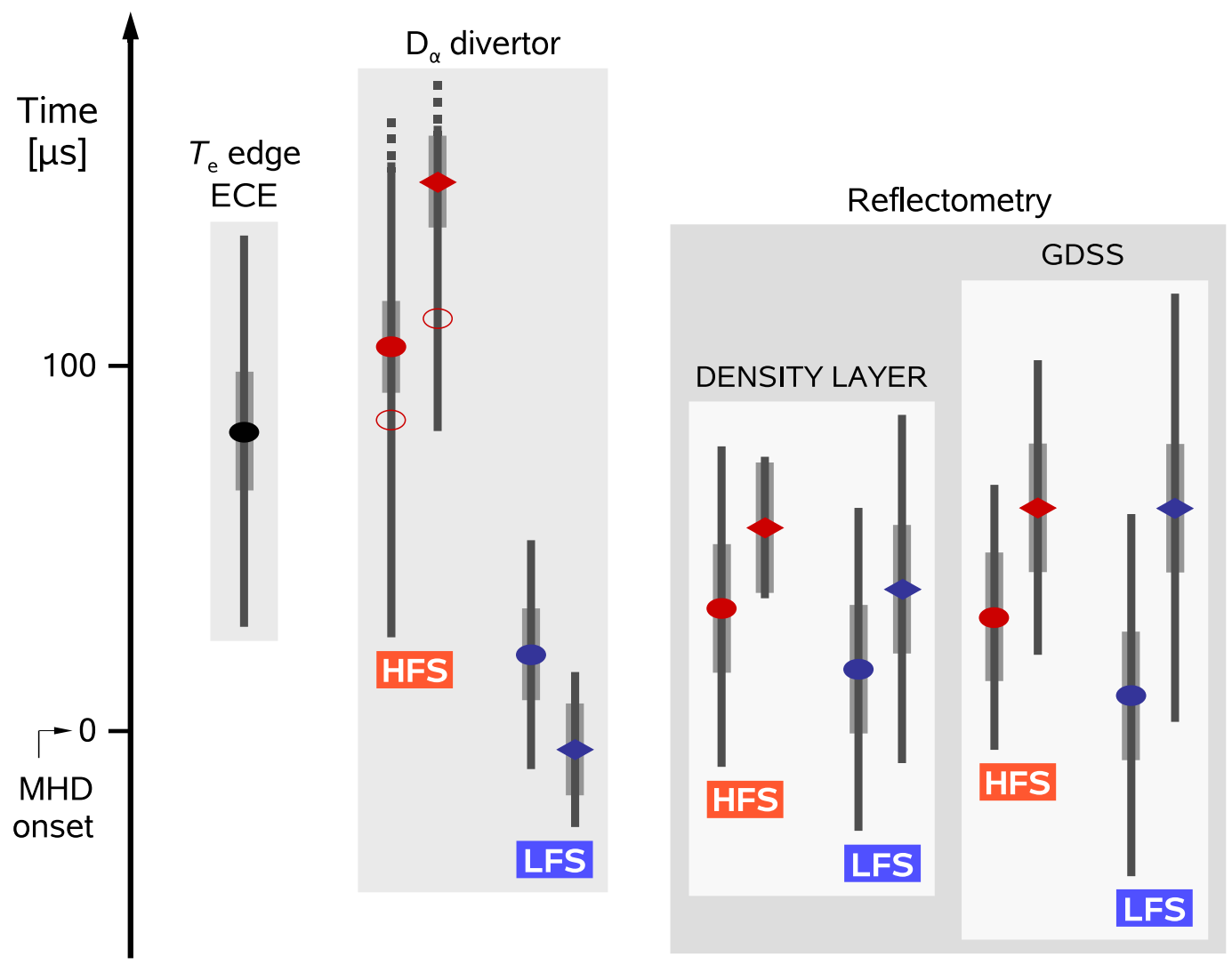

Figure 9. ELM ( $\bullet$ spontaneous and - triggered) onset times times, at HFS and LFS, evaluated from ECE, divertor $\mathrm{D}_{\alpha}$ signal and reflectometry, using different analysis techniques. Further details in the text.

reference set of pick-up coils (Kocsis et al 2007). Full circles and diamonds represent averaged values for the diagnostic signal onset for spontaneous and triggered events, respectively. The time axis is vertically upward and time equal to zero corresponds to the MHD marker. Thin black lines represent the data scatter (FWHM), thick grey bars indicate the diagnostic resolution. It is seen that, roughly speaking, the barrier has started to collapse within less than $100 \mu$ s after the first indication of the ELM onset. 
Looking in more detail, the rise of the temperature perturbation and the inner divertor radiation are clearly delayed to the ELM onset due to the gradual action of the barrier collapse on the temperature profile and the resulting subsequent pressure pulse arriving in the divertor (strictly this cannot be claimed for the $T_{\mathrm{e}}$ evolution of the triggered ELMs in our data set since not sufficient data were available and the pellet induced cooling causes less signal-to-noise ratio, but analysis of additional cases indicates both types are rather similar). There is, however, at best a slight delay of the density profile collapse for spontaneous ELMs and a somewhat larger one for the triggered ones - the fuelling effect partially masks the profile collapse by delaying the ELM onset detection, as described in section 4.2. In addition, the LFS density seems to respond a bit more promptly. To understand these results in more detail, we have to take into account the communication from some local, initial seed perturbation around the torus to the respective diagnostic locations, as discussed earlier for the shear Alfvén wave. The fast electron cooling wave (traveling on a similar time scale as the Alfvén wave) is expected for the pellet case nearly in coincidence with the magnetic onset. On the other hand, the early onset of the density perturbations at HFS and LFS, surprising at first glance, might be qualitatively consistent with the much slower ion sound wave communication along field lines. For the pellet case, the field line length from the HFS injection point to the respective HFS and LFS reflectometry antennae typically corresponds to one toroidal revolution, i.e. a delay of the order of $100 \mu \mathrm{s}$ for the ions arrival (for $q_{95} \approx 5$ ). Keeping in mind that the pellet ablation also starts about $100 \mu$ s before the ELM onset, it is troublesome to clearly fully disentangle pellet deposition and ELM effects. In case of a spontaneous ELM the reason for approximate coincidence of density drop and magnetic ELM onset signal is less clear.

The ELM onset time has been also verified analyzing the temporal evolution of the group delay distribution corresponding to a choosen probing frequency $F$. This data is directly extracted from the group delay spectrograms. An example for a spontaneous ELM (plasma discharge \#19821, time interval between 3.046270 and $3.047215 \mathrm{~s}$ ) is shown in figure 10 for the density layers $n_{\mathrm{e}}=4.2 \cdot 10^{19} \mathrm{~m}^{-3}$ (HFS) and $n_{\mathrm{e}}=1.4 \cdot 10^{19} \mathrm{~m}^{-3}$ (LFS). The normalized group delay distribution curves have been shifted along the $\tau$-axis to move the main peak of each curve to the zero value the plots in figure 10 represent the normalized and shifted group delay distribution curves. In both HFS and LFS, the group delay distribution remains almost constant during the unperturbed phase before the ELM (continuous black lines). At the HFS (see figure 10(a)), an increase of the main peak width (purple line) determines the ELM onset time. Secondary peaks appear after the ELM onset (dashed black lines) due to the presence of density fluctuations (see section 3.1). At the LFS (see figure 10(b)), the ELM onset time corresponds to a curve (purple line) having only positive values and characterized by the presence of several secondary peaks. At the ELM onset (for this particular ELM event) the main peak of the group delay distribution moved towards lower $\tau$ values, close to zero - this explains the absence of negative values once the normalized distribution curve is shifted. $35 \mu$ s after the ELM onset (dashed red line) the 


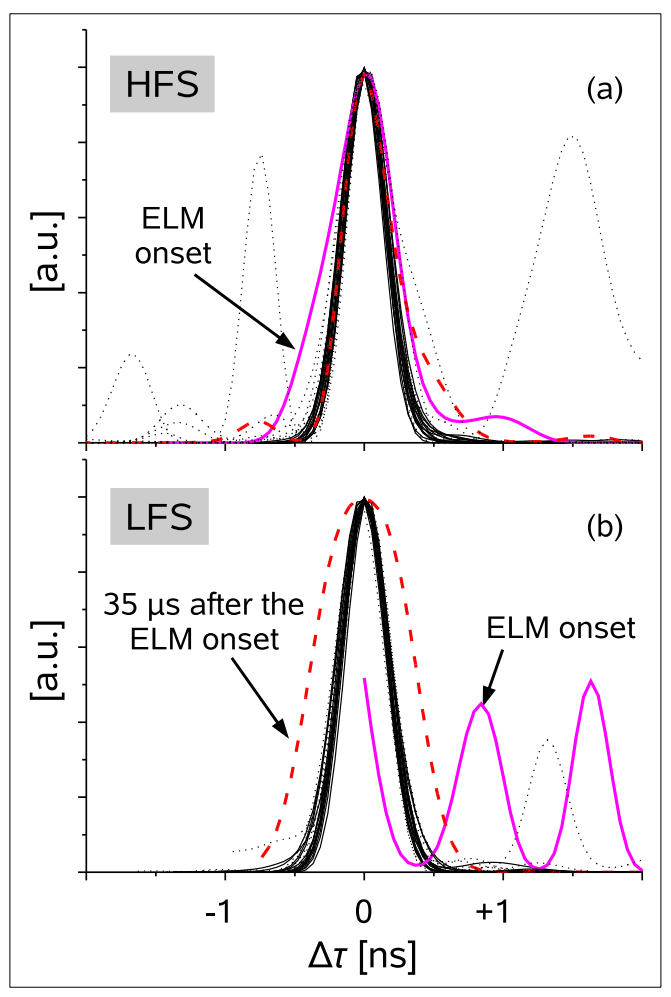

Figure 10. ELM onset time detection from the group delay distribution width at (a) HFS, for the density layer $n_{\mathrm{e}}=4.2 \cdot 10^{19} \mathrm{~m}^{-3}$, and (b) LFS, for $n_{\mathrm{e}}=1.4 \cdot 10^{19} \mathrm{~m}^{-3}$ (example from \#19821). All ELM phases are shown: unperturbed phase before the ELM onset (continuous black lines), ELM onset (purple line) and phase after the ELM onset (dashed black lines). The phase just $35 \mu$ s after the ELM onset is also shown (dashed red line).

main peak exhibits a larger width compared with the unperturbed phase. Independently from the ELM analyzed, it is always possible to select an appropriate density layer where the group delay distribution change radically at the ELM onset. This variation is represented by an increase of the main peak width or by the appearence of secondary peaks. Several ELMs, spontaneous and triggered, have been checked and results confirm the onset times detected by applying the GDSS and the DENSITY LAYER techniques. Due to its simplicity, and once perfected, this method can contribute to the fully automatic detection of the ELM onset.

\subsection{HFS/LFS asymmetries}

Although from the dynamics point of view both HFS and LFS density profiles respond qualitatively in a very similar way to the ELM, asymmetries are observed. From the HFS and LFS ELM onset times, computed by the GDSS and DENSITY LAYER techniques, it is possible to evaluate asymmetries. The observed delay between the HFS and the LFS (at the midplane) is defined as $\Delta t_{\mathrm{LFS} / \mathrm{HFS}} \equiv t_{0}^{\mathrm{HFS}}-t_{0}^{\mathrm{LFS}}$, where $t_{0}^{\mathrm{HFS}}$ and $t_{0}^{\mathrm{LFS}}$ are the ELM onset times (derived from reflectometry measurements) at the HFS and at the 
LFS, respectively. Positive values of $\Delta t_{\mathrm{LFS} / \mathrm{HFS}}$ represent HFS density profile collapse delayed respect to the LFS. The GDSS and the DENSITY LAYERS techniques gave quite similar results for spontaneous ELMs, with $\left\langle\Delta t_{\mathrm{LFS} / \mathrm{HFS}}\right\rangle=20.7 \pm 45.4 \mu \mathrm{s}$ for the GDSS technique and $\left\langle\Delta t_{\mathrm{LFS} / \mathrm{HFS}}\right\rangle=15.9 \pm 27.0 \mu \mathrm{s}$ for the DENSITY LAYER technique, where $\langle\ldots\rangle$ is the average value over the data set and the error is given by the standard deviation $\sigma$. Figure 11(a) presents histograms of the observed $\Delta t_{\text {LFS/HFS }}$ using the data from the GDSS technique. This statistical delay is zero within the temporal diagnostic resolution $(35 \mu \mathrm{s})$.
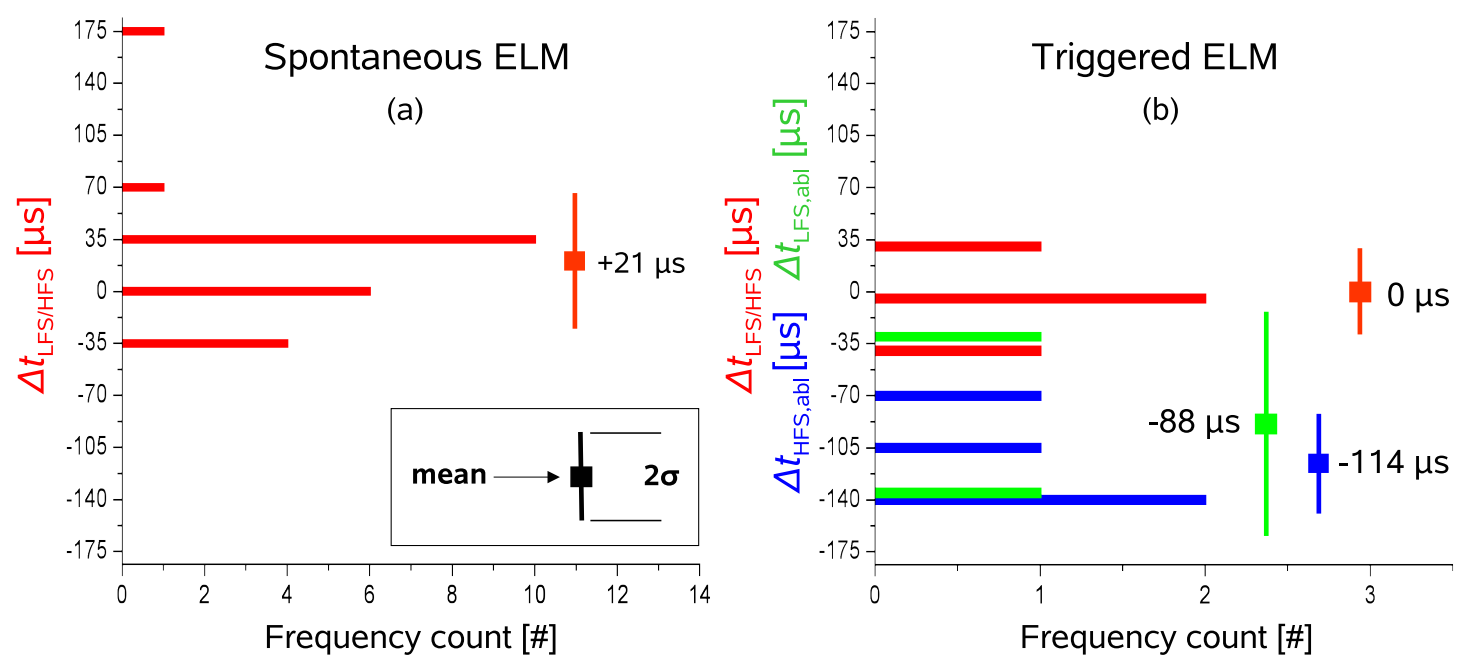

Figure 11. Histograms, mean value and standard deviation, of the ELM onset delay between HFS and LFS $\Delta t_{\text {LFS/HFS }}$ for (a) spontaneous and (b) triggered ELMs (data from the GDSS technique). For the triggered ELM the HFS and LFS pellet ablation statistics are also shown (to separate the different components, histograms are slightly vertically shifted).

A previous study (Nunes et al 2004) reported a clear HFS/LFS asymmetry with respect to edge density collapse for spontaneous ELMs. The asymmetry was attributed to the time $\tau_{\|}$needed for the ions to stream, with ion sound speed $c_{\mathrm{s}}$, from the LFS to the HFS midplane. The ion streaming time is given by $\tau_{\|}=\pi R q / c_{\mathrm{s} \text {,ped }}$, where $c_{\mathrm{s} \text {,ped }} \sim \sqrt{\left(T_{\mathrm{e} \text {,ped }}+T_{\mathrm{i} \text {,ped }}\right) / m_{\mathrm{i}}}$ and $\pi R q$ is the connection lenght inside the separatrix from the LFS to the HFS midplane across the plasma top (opposite to the X-point). For our study we have chosen a discharge configuration virtually identical to one for which Nunes reported $\tau_{\|} \sim 100 \mu \mathrm{s}$. Thus the predicted and expected HFS/LFS asymmetry was well beyond the temporal resolution $(35 \mu \mathrm{s})$ of the reflectometry system. Hence, this configuration was expected to display a clearly asymmetric behaviour for spontaneous ELMs yielding a suitable reference for triggered ELMs. However, the reported and expected asymmetry for the spontaneous ELMs could not be reproduced. Within the temporal resolution of our measurements, a significant delay between edge density collapse at LFS and HFS could be found neither for spontaneous nor for triggered ELMs.

For further clarification, we analyzed the temporal evolution of the $\mathrm{D}_{\alpha}$ radiation 
from the inner and outer divertor strike points as well, thought to correlate approximately with the ELM released plasma flux arriving at the divertor target plates. The observed average ELM onset time $(t=0$ again defined relative to the MHD marker) for the outer divertor results $\left\langle t_{0, D_{\alpha}}^{\mathrm{LFS}}\right\rangle=21.0 \pm 31.6 \mu \mathrm{s}$, while for the inner divertor we have $\left\langle t_{0, \mathrm{D}_{\alpha}}^{\mathrm{HFS}}\right\rangle=105.5 \pm 79.8 \mu \mathrm{s}$ (see figure 9 ). The $\mathrm{D}_{\alpha}$ signal at the HFS does not increase as rapidly as at LFS. The abrupt increase of the signal (that has been used to define the ELM onset from the $\mathrm{D}_{\alpha}$ signal) is often preceded by a mild increase, just above the baseline signal (see figure 12). Considering this first increase of the signal as the

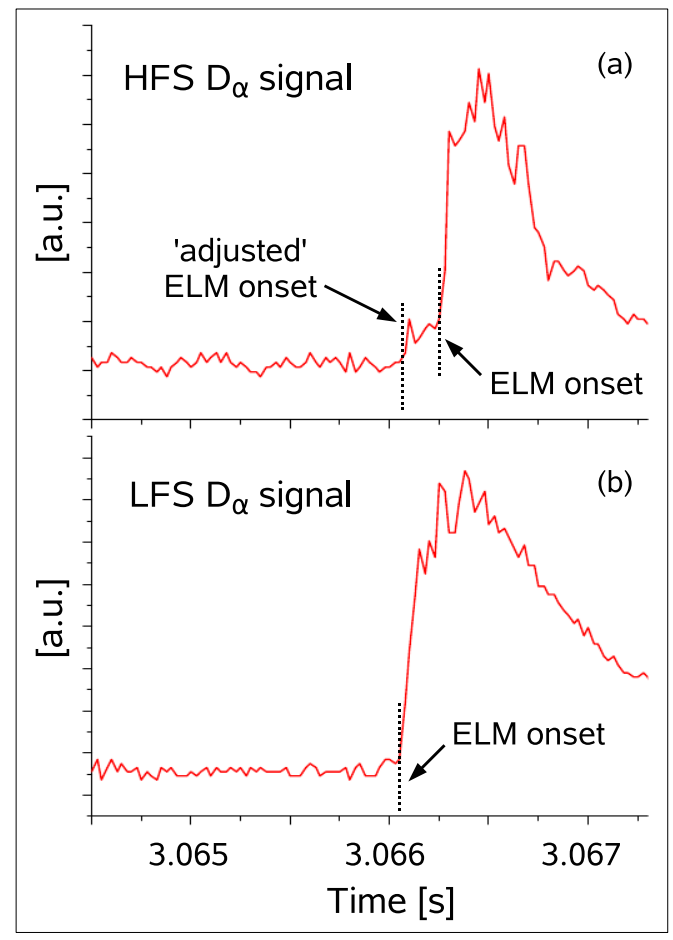

Figure 12. ELM onset time detection from divertor $\mathrm{D}_{\alpha}$ signals at (a) HFS and (b) LFS. To clearly show the two HFS onsets, an example from \#19821, representing one of the larger gaps in the analyzed data set between the two onsets, has been chosen.

ELM onset we observe an 'adjusted' $\left\langle t_{0, \mathrm{D}_{\alpha}}^{\mathrm{HFS}}\right\rangle=85.7 \pm 67.3 \mu$ s (indicated by an open circle in figure 9). It is clear that the onset of the ELM impact on the inner and outer divertor is difficult to resolve from the $\mathrm{D}_{\alpha}$ radiation alone since this parameter depends on various parameters. Furthermore, the details of the onset such as the preceding gentle increase at the HFS might be caused by non-thermal electrons or filamentary transport. For a precise interpretation more dedicated local measurements and observation with fast camera systems would be required - means not at hand during the experiments reported here. Nevertheless, a distinct delay between LFS and HFS impact is obvious, as can be realized as well when comparing the timing of the peak $\mathrm{D}_{\alpha}$ radiation which would be assumed to correlate with the maximum of the ion flux released by the ELM. Looking at the delay between HFS and LFS (at the lower divertor), measurements with $\mathrm{D}_{\alpha}$ are more or less in agreement with Nunes' expectations for this discharge configuration 
$\left(\Delta t_{\mathrm{LFS} / \mathrm{HFS}} \sim 100 \mu \mathrm{s}\right)$ being $\left\langle\Delta t_{\mathrm{LFS} / \mathrm{HFS}, \mathrm{D}_{\alpha}}\right\rangle=84.6 \pm 76.3 \mu \mathrm{s}$. It should be noted that in Nunes' work the aspects of this delay were not thoroughly discussed. Despite the different connection lengths, the delay at the lower divertor was of the same order of magnitude of the one measured at the midplane by reflectometry. For completeness of our study, we also report the onset time comparison between the two diagnostics. For the ELM onset detection at the LFS the difference between the reflectometry $\left(\left\langle t_{0}^{\mathrm{LFS}}\right\rangle=9.8 \mu \mathrm{s}\right)$ and the $\mathrm{D}_{\alpha}$ measurements is very small, of $\approx 10 \mu \mathrm{s}$, while at the HFS the $\mathrm{D}_{\alpha}$ "sees" the ELM much later than the reflectometry $\left(\left\langle t_{0}^{\mathrm{HFS}}\right\rangle=30.5 \mu \mathrm{s}\right)$ with a delay of $\approx 75 \mu$ s (this discrepancy is reduced to $\approx 55 \mu$ s using the 'adjusted' value of the $\mathrm{D}_{\alpha}$ ELM onset time).

Obviously, despite the fact that it was possible to re-establish one of the plasma configurations used in the Nunes' study, at least in terms of global plasma features, the ELM and edge dynamics look quite different. In our study we could not detect a significant HFS/LFS asymmetry of edge barrier collapse. Due to the generally shorter delay times derived, ion transport seems not to play a significant role anymore. This can be concluded also from the delays of ELM impact to the inner and outer divertor strike points, with respect to the ELM MHD onset, and the corresponding connection lengths. Assuming streaming ions carrying the energy with an ion temperature of about $1 \mathrm{keV}$, the about $45 \mathrm{~m}$ connection length (calculated $5 \mathrm{~mm}$ outside the separatrix) from the LFS mid plane - where the main particle out flux is supposed - to the inner divertor strike point define a time delay of $\approx 160 \mu \mathrm{s}$. The observed time delay between these two points is instead of $\approx 90 \mu \mathrm{s}$. To justify this delay an ion temperature of $\approx 2.4 \mathrm{keV}$ is required (even a higher temperature is required using the 'adjusted' value of the $\mathrm{D}_{\alpha}$ ELM onset time). That the ion temperature in the ELM filament outside the separatrix would exceed the temperature at the pedestal top by more than a factor of 2 seems very unlikely. This issue cannot be fully resolved in the context of this study but requires a further dedicated investigation expanding the technique applied here just for a single configuration to a full scan of $q$ as in Nunes' study. A corresponding proposal has been put forward for introduction into the ASDEX Upgrade program.

Despite the fact that for the triggered ELMs the statistics are very poor (only four ELMs), we present the histograms of the observed $\Delta t_{\mathrm{LFS} / \mathrm{HFS}}$ in figure $11(\mathrm{~b})$, for the GDSS technique, with $\left\langle\Delta t_{\mathrm{LFS} / \mathrm{HFS}}\right\rangle=0.0 \pm 28.6 \mu \mathrm{s}$. These first results, that need to be extended to more ELMs in future studies, seem very similar to the spontaneous ELMs $\left(\left\langle\Delta t_{\mathrm{LFS} / \mathrm{HFS}}\right\rangle=20.7 \pm 45.4 \mu \mathrm{s}\right)$. In the same figure, together with the $\left\langle\Delta t_{\mathrm{LFS} / \mathrm{HFS}}\right\rangle$, the average ablation time delays for triggered ELMs at HFS $\left\langle\Delta t_{\mathrm{HFS}, \text { abl }}\right\rangle$ $\left(\Delta t_{\mathrm{HFS}, \mathrm{abl}} \equiv t_{\mathrm{abl}}^{\mathrm{HFS}}-t_{0}^{\mathrm{LFS}}\right)$ and at LFS $\left\langle\Delta t_{\mathrm{LFS}, \mathrm{abl}}\right\rangle\left(\Delta t_{\mathrm{LFS}, \mathrm{abl}} \equiv t_{\mathrm{abl}}^{\mathrm{LFS}}-t_{0}^{\mathrm{LFS}}\right)$ are shown, where $t_{\mathrm{abl}}^{\mathrm{HFS}}$ and $t_{\mathrm{abl}}^{\mathrm{LFS}}$ are the observed ablation times at HFS and LFS respectively. The values $\left\langle\Delta t_{\mathrm{HFS}, \mathrm{abl}}\right\rangle=-113.8 \pm 33.5 \mu \mathrm{s}$ and $\left\langle\Delta t_{\mathrm{LFS}, \mathrm{abl}}\right\rangle=-87.5 \pm 74.3 \mu \mathrm{s}$ match very well the value observed by the camera (Kocsis et al 2007). In their study the HFS ablation was detected $\approx 70 \mu$ s before the MHD time marker, here we found a very close result, within the temporal diagnostic resolution, with $\left\langle t_{\mathrm{abl}}^{\mathrm{HFS}}\right\rangle \approx 53 \mu \mathrm{s}$. As for the HFS/LFS asymmetry of the ELM onset, also the average delay $(\approx 26 \mu \mathrm{s})$ of the LFS ablation time, with respect 
to the HFS ablation time, does not seem consistent with a parallel ion sound speed flux of the pellet ablated density from the HFS to the LFS inside the closed flux surface. In fact, assuming a pedestal temperature of $\approx 700 \mathrm{eV}$, typical for ELMy H-mode discharges (Gál et al 2008), the expected delay should be a factor of three larger being $\tau_{\|} \approx 85 \mu \mathrm{s}$. As discussed at the end of section 4.2, a rigorous evaluation of this phenomenon is very complicated.

In conclusion there is no clear evidence of large HFS/LFS asymmetries in the ELM onset for both types of ELMs, while HFS/LFS asymmetries in pellet ablation and particle deposition are observed, however mainly at the plasma pedestal top. One interpretation of these results is that the MHD aspect of the ELM instability is flattening the gradient inside the separatrix all the way to the HFS. This does not necessarily imply that density is also lost across the separatrix at the HFS but is counter to the usual view of the peeling-ballooning instability acting primarily at the LFS.

\section{Summary}

The above study has shown that the GDSS technique has the advantage of a 'direct' (still depending on the density path) and simple evaluation (fully automatic). As it does not require the evaluation of the density profile, it is independent of any initialization procedure (O-mode operation only) or errors associated with X-mode (when X-mode is utilized to complement O-mode at the very edge). The technique is very useful to track fast local profile changes and it can provide also the localization (in space and time) of density perturbations associated with fast plasma events, such as ELMs. It has the advantage that it can be used even when high resolution $(25 \mu \mathrm{s})$ single sweep density profiles are distorted due to plasma turbulence. Even if at first glance, the GDSS and the DENSITY LAYER techniques seems to give very similar results in the detection of the ELM onset time for spontanueous ELMs (see figure 9), this is due to the characteristic of the analyzed data set. The group delay spectrograms for the analyzed 22 ELMs were not so highly distorted as usually occurring during an ELM event. Due to this reason, the density profiles could have been entirely reconstructed allowing the DENSITY LAYER technique to match the GDSS potentiality. A reasonable comparision between the two techniques for triggered ELMs is not possible due to the limited number of events studied. Apart from that, the GDSS technique exhibits its superiority against the DENSITY LAYER technique regarding the localization of density fluctuations. In fact, the GDSS plots still contain the full information on the density layer fluctuations that are lost once the density profiles are evaluated.

Using high temporal and spatial resolution reflectometry with simultaneous measurements from both HFS and LFS, a detailed characterization of the ELM dynamics has been carried out from the temporal evolution of density layers features by means of the GDSS analysis. The evaluation of the ELM onset time is in good agreement with MHD measurements.

Apart from small differences in the general behaviour, spontaneous and triggered 
ELMs have almost identical characteristics. The profile flattening due to the edge barrier collapse is qualitatively similar for both types of ELMs except for the density profile steepening due to the fuelling effect of the deuterium pellets. For triggered ELMs it is not possible to evaluate precisely the ELM onset time since the fuelling effect masks the density barrier collapse during its initial phase. However, the ELM onset times are very close for both types of ELMs. Steepening and fuelling differences have been detected for triggered ELMs between HFS and LFS - the pellet induced effects on the density profile appear delayed and with reduced intensity at the LFS compared with HFS. The less pronounced fuelling effects observed at the LFS can also be due to the higher level of turbulence present there. For the plasma discharges analyzed here there is no clear evidence of HFS/LFS asymmetries in the spontaneous ELM onset time. This seems to contradict earlier studies reporting significant asymmetries for a similar plasma configuration. The reason for this is as yet unresolved but experiments are in preparation for an extended comparison, expanding our method to a wider range of edge configurations.

Reflectometry as well as divertor particle flux measurements are located at two fixed specific locations, while it should not be forgotten that ELMs consist of a burst of extremely fast 3D, highly non-linear helical instabilities (Neuhauser et al 2008).

Triggered ELMs seem to exhibit the same behaviour as spontaneous ones. However to confirm our preliminary results statistically this analysis has to be extended to a higher number of pellet triggered ELMs. Furthermore, as mentioned by Neuhauser, although the pellet clearly acts as the principal ELM trigger, the dominant physical mechanisms still remain open, since the ELMs start at random toroidal phase relative to the pellet launch position.

\section{Acknowledgments}

L. F. would like to thank Dr. J. Neuhauser for the fruitful discussions, Dr. T. Eich and Dr. C. Angioni for the estimation of the connection lengths and acknowledges useful comments of Dr. S. Ratynskaia. This work has been carried out in the frame of the Contract of Association between the European Atomic Energy Community and Instituto Superior Técnico (IST) and of the Contract of Associated Laboratory between Fundação para a Ciência e Tecnologia (FCT) and IST. The content of the publication is the sole responsibility of the authors and it does not necessarily represent the views of the Commission of the European Union or FCT or their services.

\section{References}

ASDEX Upgrade Team 2003 Fusion Sci. Technol. 44569

Budden K G 1988 The propagation of radio waves: the theory of radio waves of low power in the ionosphere and magnetosphere Paperback

Connor J W, Hastie R J, Wilson H R and Miller R L 1998 Phys. Plasmas 52687 
Fattorini L, Lang P T, Manso M E, Kálvin S, Kocsis G and the ASDEX Upgrade Team 2005 Proc. 32nd EPS Conf. on Plasma Physics (Tarragona 2005) P5.089

Federici F 2006 Phys. Scr. T124 1

Gál K, Belonohy É, Kocsis G, Lang P T, Veres G and the ASDEX Upgrade Team 2008 Nucl. Fusion 48085005

Ginzburg V L 1964 The propagation of electromagnetic waves in plasma Pergamon

Kocsis G, Kálvin S, Lang P T, Maraschek M, Neuhauser J, Schneider W, Szepesi T and the ASDEX Upgrade Team 2007 Nucl. Fusion 471166

Lang P T, Cierpka P, Gehre O, Reich M, Wittmann C, Lorenz A, Frigione D, Kálvin S and Kocsis G 2003 Rev. Sci. Instrum. 743974

Lang P T et al 2004 Nucl. Fusion 44665

Lang P T et al 2005 Proc. 32nd EPS Conf. on Plasma Physics (Tarragona 2005) 04.003

Lang $\mathrm{P}$ T et al 2005a Nucl. Fusion 45502

Lang P T, Fattorini L, Kálvin S, Kocsis G, Manso M E, Maraschek M, Neuhauser J, Suttrop W and the ASDEX Upgrade Team 2006 Proc. 33rd EPS Conf. on Plasma Physics (Roma 2006) P2.150

Manso M E, Santos J, Nunes I, Kurzan B, Serra F, Silva A and Suttrop W 1997 Proc. 24th EPS Conf. on Plasma Physics (Berchtesgaden 1997) P4.1489

Neuhauser J, Bosch H S, Coster D, Herrmann A and Kallenbach A 2003 Fusion Sci. Technol. 44659

Neuhauser J et al 2008 Nucl. Fusion 48045005

Nunes I, Conway G D, Loarte A, Manso M E, Serra F, Suttrop W and the CFN and ASDEX Upgrade Teams 2004 Nucl. Fusion 44883

Oyama N, Shinohara K, Kamada Y, Miura Y, Oikawa T and Takeji S 2001 Plasma Phys. Control. Fusion 43717

Santos J, Manso M E, Nunes F, Nunes I, Silva A and Varela P 1997 Proc. 3rd International Workshop on Microwave Reflectometry for Fusion Plasma Diagnostics (Madrid 1997) 55

Silva A, Varela P, Cupido L, Manso M E, Meneses L, Guimarães L, Conway G D and the ASDEX Upgrade Team 2005 Proc. 7th International Workshop on Microwave Reflectometry for Fusion Plasma Diagnostics (Garching 2005) 3

Swanson D G 1989 Plasma waves Academic Press

Varela P, Manso M E, Silva A, Fernandes J and Silva F 1995 Rev. Sci. Instrum. 664937

Varela P, Manso M E, Nunes I, Santos J, Nunes F, Silva A and Silva F 1999 Rev. Sci. Instrum. 70 1060

Varela P, Manso M E, Silva A, the CFN Team and the ASDEX Upgarde Team 2006 Nucl. Fusion 46 $\mathrm{S} 693$

Wilson H R, Connor J W, Field A R, Fielding S J, Hastie R J, Miller R L and Taylor J B 2000 Nucl. Fusion $\mathbf{4 0} 713$

Zeng L, Wang G, Doyle E J, Rhodes T L, Peebles W A, McKee G R, Fonck R, Burrel K H, Fenstermacher M E, Boedo J and Moyer R 2004 Plasma Phys. Control. Fusion 46 A121

Zeng L, Wang G, Doyle E J, Rhodes T L, Peebles W A, Fenstermacher M E, Evans T E and Moyer R A 2005 J. Nucl. Mater. 337742 